ACTA ARITHMETICA

XXXII (1977)

\title{
Generalization of some theorems on sets of multiples and primitive sequences
}

by

W. KLotz (Clansthal)

1. Introduction. The main results of this paper are generalizations of a theorem of Besicovitch on primitive sequences and of a theorem of Davenport and Erdös on sets of multiples. For these theorems and a survey of related results we refer to the final chapter of Halberstam and Roth [3].

By a system $\sigma$ we mean a non-empty set of finite, non-empty sets of positive integers. The system $\sigma$ is called homogeneous, if for each $n \in N$ (set of positive integers)

$$
S \in \sigma \text { implies } n S=\{n s: s \in S\} \in \sigma .
$$

The set $A \subset N$ is said to be $\sigma$-free, if it does not contain a subset belonging to $\sigma$. For a given homogeneous system $\sigma$ we discuss the question of the 'greatest possible density' a $\sigma$-free set may have. We investigate natural densities and logarithmic densities of $\sigma$-free sets.

The author wishes to acknowledge his debt to the valuable advice of Professor E. Wirsing.

2. Natural densities of $\sigma$-free sets. First we introduce some notations. For real numbers $\alpha, \beta$ we define the interval $[\alpha, \beta]=\{n: n \in N, \alpha \leqslant n$ $\leqslant \beta$. If $A$ is a finite set, then $|A|$ denotes the number of elements in $A$. The counting function of $A \subset N$ is $A(n)=|A \cap[1, n]|$. The limit $d(A)$ $=\lim A(n) / n$, if it exists, is called the natural density of $A . '$ The lower and upper natural densities $d(A)$ and $\bar{d}(A)$ are defined by the liminf and limsup of the same expression. The system $\sigma$ is characterized by

$$
\tau_{\sigma}(n)=\max \{A(n): A \sigma \text {-free }\}
$$

$$
\underline{\tau}_{\sigma}=\liminf _{n \rightarrow \infty} \tau_{\sigma}(n) / n, \quad \bar{\tau}_{\sigma}=\limsup _{n \rightarrow \infty} \tau_{\sigma}(n) / n .
$$

If $\underline{\tau}_{\sigma}=\bar{\tau}_{\sigma}$ let $\tau_{\sigma}=\underline{\tau}_{\sigma}=\bar{\tau}_{\sigma}$. Furthermore, we défine

$$
\underline{d}(\sigma)=\sup \{\underline{d}(A): A \text {-free }\}, \quad \bar{d}(\sigma)=\sup \{\bar{d}(A): A \sigma \text {-free }\} .
$$

If $\underline{d}(\sigma)$ and $\bar{d}(\sigma)$ coincide, the common value is denoted by $d(\sigma)$. 
Every system $\sigma_{0}$ generates a homogeneous system $\sigma$,

$$
\sigma=N \sigma_{0}=\left\{T: T=n S, n \in N, S \in \sigma_{0}\right\} .
$$

The investigation of a homogeneous system is facilitated by a small generating system.

THEOREM 1. Suppose that the homogeneous system $\sigma$ is generated by $\sigma_{0}=\left\{S_{1}, S_{2}, \ldots\right\}$. Let $a_{1}, a_{2}, \ldots, a_{k}$ be coprime integers greater than 1 and

$$
U=\left\{u: u=a_{1}^{r_{1}} a_{2}^{r_{2}} \ldots a_{k}^{r_{1}}, r_{i} \in\{0\} \cup N\right\} .
$$

If $S_{i} \subset U$ for each $i$ then $\tau_{\sigma}$ exists and

$$
\tau_{\sigma}(n)=\tau_{\sigma} n+O\left(\log ^{k} n\right)
$$

Here $\tau_{\sigma}$ is less than 1. There is a o-free set $A$ with $\bar{d}(A)=\tau_{\sigma}$.

Proof. Denote by $V$ the sequence of positive integers which are not a multiple of any of the numbers $a_{j}$. It is well-known that

$$
\text { (1) } \quad V(n)=n d(V)+O(1), \quad \text { where } \quad a(V)=\prod_{j=1}^{k}\left(1-\frac{1}{a_{j}}\right) \text {. }
$$

We have

$$
\sum_{u \in U} \frac{1}{u}=\frac{1}{d(V)} \text { and } \quad U(n) \leqslant\left(1+\frac{\log n}{\log 2}\right)^{k}
$$

Every positive integer has a unique representation of the form $u v, u \in U$, $v \in V$. Therefore, it follows from (1) and (2)

$$
\begin{gathered}
n=\sum_{u \leqslant n} V\left(\frac{n}{u}\right)=n d(V) \sum_{u \leqslant n} \frac{1}{u}+O(U(n)), \\
\sum_{u>n} \frac{1}{u}=o\left(\frac{U(n)}{n}\right)=O\left(\frac{\log ^{k} n}{n}\right),
\end{gathered}
$$

where summation is taken over the numbers $u \in U$.

If we define

$$
\tau_{\sigma}^{U}(n)=\max \{A(n): A \subset U, A \sigma \text {-free }\},
$$

and if $R$ denotes the unique subset of $U$ lawving the counting function $R(n)=\tau_{\sigma}^{U}(n)$ then

Thus, by (1),

$$
\tau_{\sigma}(n)=\sum_{v \in V} \tau_{\sigma}^{U}\left(\frac{n}{v}\right)=\sum_{v \in \bar{V}} R\left(\frac{n}{v}\right)
$$

$$
\tau_{\sigma}(n)=\sum_{r \in R} V\left(\frac{n}{r}\right)=n d(V) \sum \frac{1}{r}-n d(V) \sum_{r>n} \frac{1}{r}+O(R(n)),
$$

where summation is over $r \in R$. By $R \subset U$, it now follows from (2) and (3) that

(4) $\quad \tau_{\sigma}(n)=\tau_{\sigma} n+O\left(\log ^{k} n\right)$, where $\tau_{\sigma}=a(V) \sum_{r \in R} \frac{1}{r}$.

Since $\sigma$ and the sets $S_{i}$ are non-empty by definition, $R$ is a proper subset of $U$. We have

$$
\sum_{r \in R} \frac{1}{r}<\sum_{u \in U} \frac{1}{u}=\frac{1}{d(V)}
$$

whence $\tau_{\sigma}<1$. The existence of a $\sigma$-free set $A$ with $\bar{d}(A)=\tau_{\sigma}$ is ensured by the following lemma.

LEMma 1. Suppose that the homogeneous system $\sigma$ is generated by $\sigma_{0}=\left\{S_{1}, S_{2}, \ldots\right\}$. If $M=\left\{z: z=\max S_{i}, S_{i} \in \sigma_{0}\right\}$ has natural density 0 , then there is a $\sigma$-free set $A$ with $\bar{d}(A)=\bar{\tau}_{\sigma}$.

Pro of. Let $\varepsilon_{j}(j=1,2, \ldots)$ be positive numbers satisfying $0<\varepsilon_{j}<1$ and $\lim \varepsilon_{j}=0$. There is a sequence of integers $x_{j}$ starting with $x_{0}=0$ and having the following properties for $j>0$.

$$
\begin{aligned}
& \text { (a) } x_{j}>\frac{1}{\varepsilon_{j}} x_{j-1}, \\
& \text { (b) } \tau_{\sigma}\left(x_{j}\right)>\left(\bar{\tau}_{\sigma}-\varepsilon_{j}\right) x_{j}, \\
& \text { (c) if } T_{j-1}=\left\{m n: m \in M, n \in\left[1, x_{j-1}\right]\right\} \text { then } T_{j-1}\left(x_{j}\right)<\varepsilon_{j} x_{j} .
\end{aligned}
$$

Let $A_{j}^{\prime}$ be a $\sigma$-free set in $\left[1, x_{j}\right]$ with $\left|A_{j}^{\prime}\right|>\left(\bar{\tau}_{\sigma}-\varepsilon_{j}\right) x_{j}$. Using the notation $B \neg C=\{z: z \in B, z \notin C\}$ we define

$$
\left.A_{j}=A_{j}^{\prime}\right\urcorner\left(\left[1, x_{j-1}\right] \cup T_{j-1}\right), \quad A=\bigcup_{j=1}^{\infty} A_{j} .
$$

The sets $A_{j}$ are disjoint and $\sigma$-free. From (a), (b), (c) we obtain

$$
A\left(x_{j}\right) \geqslant A_{j}\left(x_{j}\right)>\left(\bar{\tau}_{\sigma}-3 \varepsilon_{j}\right) x_{j},
$$

hence $\bar{d}(A) \geqslant \bar{\tau}_{\sigma}$. Assume now that $A$ contains a set $n S_{i}$. Let $d=\min \cdot S_{i}$ and $D=\max S_{i}$. Since the sets $A_{j}$ are $\sigma$-free, we must have

$$
n d \in A_{T^{\prime}}, \quad n D \in A_{\alpha}, \quad k<q \text {. }
$$

From $n \leqslant x_{\eta_{6}} \leqslant x_{q-1}$ and $D \in M$ follows $n D \in T_{q-1}$, which contradicts the definition of $A_{q}$. Therefore, $A$ is $\sigma$-free.

LEMma 2. Suppose that the homogeneous system $\sigma$ is generated. by $\sigma_{0}=\left\{S_{1}, S_{2}, \ldots\right\}$. Let $d_{j}=\min S_{j}$ and $D_{j}=\max S_{j}$. If $\lim _{j \rightarrow \infty} d_{j} / D_{j}=0$ then $\tau_{\sigma}$ exists.

Pro of. Denote by $\sigma_{j}$ the homogeneous system generated by $\left\{S_{1}, S_{2}, \ldots\right.$ $\left.\ldots, S_{j}\right\}$. By Theorem 1, the gensity $\tau_{\sigma_{j}}$ exists. Moreover, $\lim _{j \rightarrow \infty} \tau_{\sigma_{j}}=\tau$ 
exists, because $\tau_{\sigma_{j}} \geqslant \tau_{\sigma_{j+1}}$. If for positive $\varepsilon$ the integer $j$ is chosen so large that $d_{k} / D_{k}<\varepsilon$ for each $k>j$. then *

$$
\tau_{\sigma_{j}}(n)-\varepsilon n \leqslant \tau_{\sigma}(n) \leqslant \tau_{\sigma_{j}}(n), \quad \tau_{\sigma_{j}}-\varepsilon \leqslant \underline{\tau}_{\sigma} \leqslant \bar{\tau}_{\sigma} \leqslant \tau_{\sigma_{j}} .
$$

Fror $j \rightarrow \infty$ and $\varepsilon \rightarrow 0$ we obtain $\tau_{\sigma}=\bar{\tau}_{\sigma}=\tau$.

We are now going to state the announced generalization of a theorem of Besicoviteh ([3], p. 257) on primitive sequences. We denote by $N G$ the set of multiples $\{n g: n \in N, g \in G\}$.

THEOREM 2. Let the homogeneous system $\sigma$ be generated by $\sigma_{0}=\left\{S_{1}\right.$, $\left.S_{2}, \ldots\right\}$. Suppose that there is a sequence $G=\left\{g_{1}, g_{2}, \ldots\right\}$ of positive integers satisfying

(i) $S_{j} \cap G \neq \varnothing$ for each $j$

(ii) $\lim \bar{d}\left(N G_{j}\right)=0$ if $G_{j}=\left\{g_{j}, g_{j+1}, \ldots\right\}$.

Then $\tau_{\sigma}$ exists and $\bar{d}(\sigma)=\tau_{\sigma}$. Furthermore, $\tau_{\sigma}=0$ is equivalent to $\{1\} \in \sigma$, and $1 \notin G$ implies $\underline{a}(\sigma)>0$.

Proof. We make use of the following lemma which is easily deduced from an inequality of Behrend ([3], p. 263).

LEMMA 3 (Erdös [2]). If $1 \notin G$ and $\lim \bar{d}\left(N G_{j}\right)=0$ then $a(N G)$ exists and is less than 1 .

We note that $N \neg N G$ is $\sigma$-free by (i). So Lemma 3 implies $d(\sigma)>0$ if $1 \notin G$. Now suppose $1 \epsilon G$ and $G^{\prime}=G \neg\{1\}$ then

$$
\left(N \neg N G^{\prime}\right) \cap(n / 2, n]
$$

is $\sigma$-free for each $n \in N$ if $\{1\} \notin \sigma$. In this case Lemma 3 implies $\tau_{\sigma}>0$.

It remains to prove the existence of $\tau_{\sigma}$ and $\bar{d}(\sigma)=\tau_{\sigma}$. By (ii); we may assume that $G$ is finite, $G=\left\{g_{1}, \ldots, g_{t}\right\}$. Then the existence of $\tau_{a}$ follows either from Theorem 1 or Lemma 2.

LEMara 4. If $m$ is any positive real number then

$$
\lim _{x \rightarrow \infty} d(N[x / m, x])=0 .
$$

This is an immediate consequence of a theorem of Erdös ([3], p. 268). To construct a $\sigma$-free set $A$ with $\bar{d}(A) \geqslant \tau_{\sigma}-\varepsilon(0<\varepsilon<1)$, we choose

$$
m=\frac{3}{\varepsilon} g_{t}, \quad \varepsilon_{j}=\left(\frac{1}{2}\right)^{j} \frac{\varepsilon}{3} \quad(j=0,1,2, \ldots) .
$$

There is a sequence of integers $x_{j}$ starting with $x_{0}=0$ and having the following properties for $j>0$ :

(a) $x_{j}>m x_{j-1}$

(b) $\tau_{\sigma}\left(x_{j}\right)>\left(\tau_{\sigma}-\frac{1}{3} \varepsilon\right) x_{j}$

(c) if $B_{j}=N\left[x_{j} / m, x_{j}\right]$ then $a\left(B_{j}\right)<\varepsilon_{j}$ and $B_{j-1}(x)<\varepsilon_{j-1} x$ for each $x \geqslant x_{j}$.
Let $A_{j}^{\prime}$ be a $\sigma$-free set in $\left[1, x_{j}\right]$ with $\left|A_{j}^{\prime}\right|>\left(\tau_{\sigma}-\frac{1}{3} \varepsilon\right) x_{j}$. Define

$$
\left.A_{j}=A_{j}^{\prime}\right\urcorner\left(\bigcup_{i<j} B_{i} \cup\left[1, \frac{1}{3} \varepsilon x_{j}\right]\right), \quad A=\bigcup_{j=1}^{\infty} A_{j} .
$$

The sets $A_{j}$ are disjoint and $\sigma$-free. From (a), (b), (c) we obtain

$$
A\left(x_{j}\right) \geqslant A_{j}\left(x_{j}\right)>\left(\tau_{\sigma}-\frac{1}{3} \varepsilon-\frac{1}{3} \varepsilon-\sum_{i=1}^{j-1} \varepsilon_{i}\right) x_{j}>\left(\tau_{\sigma}-\varepsilon\right) x_{j},
$$

hence $\bar{d}(A) \geqslant \tau_{\sigma}-\varepsilon$. To prove that $A$ is $\sigma$-free, assume that $A$ contains a set of the form $n S_{i}, S_{i} \in \sigma_{0}$. Let $d=\min S_{i}$ and $D=\max S_{i}$. Since the sets $A_{j}$ are $\sigma$-free, we must have

$$
n d \in A_{k}, \quad n D \in A_{q}, \quad k<q .
$$

By (i), $S_{i}$ contains a number $g \in G$. Thus $n g \in A$ and, by (a), (5), and (7),

$$
n g=n d \frac{g}{d} \leqslant x_{k} g_{t}<x_{k+1} \frac{\varepsilon}{3}
$$

Now (6) implies $n g \in A_{7_{6}}$. So we have

$$
x_{k} \frac{\varepsilon}{3}<n g \leqslant x_{k}, \quad \frac{x_{k}}{m}<n \leqslant x_{k} .
$$

Therefore, $n D \in B_{k}$, which contradicts the definition of $A_{q}$.

3. Logarithmic densities of $\sigma$-free sets. For a homogeneous system $\sigma$ the natural density $d(\sigma)$ need not exist. Example 2 below shows that even for a finitely generated system $\underline{d}(\sigma)$ may be less than $\bar{d}(\sigma)$. More uniform results are obtained by considering logarithmic densities. We introduce the following logarithmic notions in analogy to the corresponding terms on natural density.

The logarithmic counting function of $A \subset N$ is $A^{*}(n)=\sum_{a \leq n} 1 / a$ (summation over $a \in A$ ). The $\operatorname{limit} \delta(A)=\lim A^{*}(n) / \log n$, if it exists, is called the logarithmic density of $A$. The lower and upper logarithmic densities $\delta(A)$ and $\delta(A)$ are defined by the liminf and limsup of the same expression. Let

$$
\begin{gathered}
\lambda_{\sigma}(n)=\max \left\{A^{*}(n): A \sigma \text {-free }\right\}, \\
\underline{\lambda}_{\sigma}=\liminf _{n \rightarrow \infty} \frac{\lambda_{\sigma}(n)}{n}, \quad \vec{\lambda}_{\sigma}=\limsup _{n \rightarrow \infty} \frac{\lambda_{\sigma}(n)}{n} .
\end{gathered}
$$

If $\underline{\lambda}_{\sigma}=\bar{\lambda}_{\sigma}$ put $\underline{\lambda}_{\sigma}=\bar{\lambda}_{\sigma}=\lambda_{\sigma}$. Define

$$
\underline{\delta}(\sigma)=\sup \{\underline{\delta}(A): A \quad \sigma \text {-free }\}, \quad \delta(\sigma)=\sup \{\delta(A): A \sigma \text {-free }\} .
$$

If $\underline{\delta}(\sigma)=\bar{\delta}(\sigma)$ denote the common value by $\delta(\sigma)$. 
We believe that on very general conditions for a homogeneous system $\delta(\sigma)$ and $\lambda_{\sigma}$ exist and coincide.

THEOREM 3. Suppose that the homogeneous system $\sigma$ is generated by $\sigma_{0}=\left\{S_{1}, S_{2}, \ldots\right\}$. Let $a_{1}, a_{2}, \ldots, a_{k}$ be coprime integers greater than 1 and

$$
U=\left\{u: u=a_{1}^{r_{1}} a_{2}^{r_{2}} \ldots a_{i}^{r_{k}}, r_{i} \in\{0\} \cup N\right\} .
$$

If $S_{i} \subset U$ for each $i$ then $\lambda_{\sigma}$ exists and

$$
\lambda_{\sigma}(n)=\lambda_{\sigma} \log n+O(\log \log n) .
$$

Furthermore, $\delta(\sigma)$ exists and $\underline{d}(\sigma)=\delta(\sigma)=\lambda_{\sigma}$. If $k=1$ there is a $\sigma$-free set $A$ satisfying

$$
A^{*}(n)=\lambda_{\sigma}(n) \quad \text { and } \quad A(n)=\lambda_{\sigma} n+O(\log n) .
$$

Proof. This proof is similar to that of Theorem 1. If we denote by $V$ the sequence of positive integers which are not a multiple of any of the numbers $a_{j}$ then

$$
\text { (8) } \quad V^{*}(n)=d(V) \log n+O(1), \quad \text { where } \quad d(V)=\prod_{j=1}^{h_{a}}\left(1-\frac{1}{a_{j}}\right) \text {. }
$$

Define

$$
\lambda_{\sigma}^{U}(n)=\max \left\{A^{*}(n): A \subset U, A \sigma \text {-free }\right\} .
$$

By (3), the limit $\lim \lambda_{\sigma}^{U}(n)=\alpha$ exists and

$$
\lambda_{\sigma}^{U}(n)=\alpha+O\left(\frac{\log ^{k} n}{n}\right)
$$

Now we have, by (8) and (9),

$$
\lambda_{\sigma}(n)=\sum_{v \in V} \frac{1}{v} \lambda_{\sigma}^{U}\left(\frac{n}{v}\right)
$$

$$
\lambda_{\sigma}(n)=\alpha d(V) \log n+O\left(\sum_{v \leq n} \frac{1}{v} \frac{(\log (n / v))^{1 c}}{n / v}\right)
$$

where $v \in V$. Let $m=\log ^{2} n$ and $f(y)=\frac{\log ^{x} y}{y}$. Assuming that $f(y)$ is strictly decreasing for $y \geqslant m$, we obtain, by (8),

$$
\left(\sum_{v \leqslant n / m}+\sum_{n / m<v \leqslant n}\right) \frac{1}{v} f\left(\frac{n}{v}\right)=O(f(m) \log n+\log m)
$$

Hence, by (10),

(11) $\quad \lambda_{\sigma}(n)=\lambda_{\sigma} \log \dot{n}+O(\log \log n), \quad$ where $\quad \lambda_{\sigma}=\alpha d(V)$.
To prove $d(\sigma)=\delta(\sigma)=\lambda_{\sigma}$, we construct a $\sigma$-free set $A$ with natural densit. greater than $\lambda_{\sigma}-\varepsilon\left(0<\varepsilon<\lambda_{\sigma}\right)$. Let $U^{\prime}$ be a finite $\sigma$-free subset of $U$ satisfying

$$
\sum_{u \in U^{\prime}} \frac{1}{u}>\alpha-\varepsilon
$$

If $A=U^{\prime} V$ then $A$ is $\sigma$-free and

$$
A(n)=\sum_{u \in U^{\prime}} V\left(\frac{n}{u}\right)=n d(V) \sum_{u \in U^{\prime}} \frac{1}{u}+O(1)
$$

Therefore, $d(A)$ exists and $d(A)>(\alpha-\varepsilon) d(\nabla)>\lambda_{\sigma}-\varepsilon$.

If $k=1$ let $a=a_{1}$ and $U_{j}=\left\{a^{0}, a^{1}, \ldots, a^{j}\right\}, U_{-1}=\varnothing$. Define $S \subset U$ by the following property:

$a^{j} \in S$. if and only if $\left(S \cap U_{j-1}\right) \cup\left\{a^{j}\right\}$ is $\sigma$-free $(j=0,1, \ldots)$.

Let $S_{j}=S \cap U_{j}$. We prove by induction that $S_{j}$ is the only $\sigma$-free set in $U_{j}$ with $S_{j}^{*}\left(a^{j}\right)=\lambda_{\sigma}^{U}\left(a^{j}\right)$. This is certainly true for $j=0$. Let it be true for $j-1(j \geqslant 1)$. Suppose now that $M$ is a $\sigma$-free subset of $U_{j}$, $M \cap U_{j-1} \neq S_{j-1}$, then

$$
M^{*}\left(a^{j}\right) \leqslant \lambda_{\sigma}^{U}\left(a^{j-1}\right)-\frac{1}{a^{j-1}}+\frac{1}{a^{j}}<S_{j}^{*}\left(a^{j}\right) .
$$

Hence $S^{*}\left(a^{j}\right)=\lambda_{\sigma}^{U}\left(a^{j}\right)$ for $j=0,1, \ldots$ If $A=S V$ then $A$ is $\sigma$-free, $A^{*}(n)=\lambda_{\sigma}(n)$, and

$$
A(n)=\sum_{s \in S} V\left(\frac{n}{s}\right)=\lambda_{\sigma} n+O(\log n), \quad \text { where } \quad \lambda_{\sigma}=d(V) \sum_{s \in S} \frac{1}{s} .
$$

LEMMA 5. Let the homogeneous system $\sigma$ be generated by $\sigma_{0}=\left\{S_{1}, S_{2}, \ldots\right\}$. Let $G=\left\{g_{1}, g_{2}, \ldots\right\}$ be a sequence of positive integers, $G_{j}=\left\{g_{j}, g_{j+1}, \ldots\right\}$, and $\sigma_{j}$ the homogeneous system generated by $\left\{S: S \in \sigma_{0}, S \cap N G_{j}=\emptyset\right\}$. Suppose

(i) $\lim \delta\left(N G_{j}\right)=0$,

(ii) $\stackrel{j \rightarrow \infty}{\delta}\left(\sigma_{j}\right)$ and $\lambda_{\sigma_{j}}$ exist and $\delta\left(\sigma_{j}\right)=\lambda_{\sigma_{j}}$ for each $j \in N$.

Then $\delta(\sigma)$ and $\lambda_{\sigma}$ exist and $\delta(\sigma)=\lambda_{\sigma}=\lim \delta\left(\sigma_{j}\right)$. If, in addition to (i) and (ii), $\lim _{j \rightarrow \infty} \bar{d}\left(N G_{j}\right)=0$ and $\underline{d}\left(\sigma_{j}\right)=\delta\left(\sigma_{j}\right)^{j \rightarrow \infty}=\lambda_{\sigma_{j}}$ for each $j \in N$ then

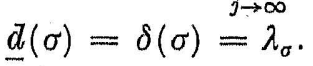

Proof. Since $\sigma_{1} \subset \sigma_{2} \subset \ldots \subset \sigma$, the limit $\lim _{j \rightarrow \infty} \lambda_{\sigma_{j}}=\lambda$ exists and $\bar{\lambda}_{\sigma} \leqslant \lambda$. Let $\varepsilon_{j}>0$ and $\lim _{j \rightarrow \infty} \varepsilon_{j}=0(j \in N)$. By (ii), there is a $\sigma_{j}$-free set $A_{j}$ with $\underline{\delta}\left(A_{j}\right)>\lambda_{\sigma_{j}}-\varepsilon_{j}$. The set $\left.A_{j}^{\prime}=A_{j}\right\urcorner N G_{j}$ is $\sigma$-free and $\underline{\delta}\left(A_{j}^{\prime}\right)>\lambda_{\sigma_{j}}-$ $-\varepsilon_{j}-\delta\left(N G_{j}\right)$. For $j \rightarrow \infty$ follows $\delta(\sigma) \geqslant \lambda$. Hence $\delta(\sigma)$ and $\bar{\lambda}_{\sigma}$ exist and $\delta(\sigma)=\lambda_{\sigma}=\lambda$.

If $\underline{d}\left(\sigma_{j}\right)=\delta\left(\sigma_{j}\right)=\lambda_{\sigma_{j}}$ and $\lim _{j \rightarrow \infty} \bar{d}\left(N G_{j}\right)=0$ then we may demand $\underline{d}\left(A_{j}\right)>\lambda_{\sigma_{j}}-\varepsilon_{j}$. Now we have $\underline{a}\left(A_{j}^{\prime}\right)>\lambda_{\sigma_{j}}-\varepsilon_{j}-\bar{d}\left(N G_{j}\right)$, and for $j \rightarrow \infty$ follows the final part of Lemma 5 . 
If $A=\left\{a_{1}, a_{2}, \ldots\right\}$ is a sequence of positive integers let $A_{j}=$ $\left\{a_{j}, a_{j+1}, \ldots\right\}$ and $\bar{A}_{j}=A \neg A_{j}$. It has been proved by Davenport and Erdös ([3], p. 258) that the logarithmic density $\delta(N A)$ exists and

$$
\begin{aligned}
& \text { Note that } \quad \underline{d}(N A)=\delta(N A)=\lim _{j \rightarrow \infty} d\left(N \bar{A}_{j}\right) . \\
& \text { (12) } \left.\quad \lim _{j \rightarrow \infty} \delta\left(N A_{j}\right\urcorner N \bar{A}_{j}\right)=0 .
\end{aligned}
$$

LEMIMA 6. Suppose that the homogeneous system $\sigma^{\prime}$ is generated by $\sigma_{0}^{\prime}=\left\{S_{1}, S_{2}, \ldots, S_{q}\right\}$. Then for any homogeneous subsystem $\sigma \subset \sigma^{\prime}$ the densities $\delta(\sigma)$ and $\lambda_{\sigma}$ exist and coincide.

Proof. Any homogeneous subsystem $\sigma$ of $\sigma^{\prime}$ is of the form Let

$\sigma=\left\{S: S=a_{i k} S_{i}, 1 \leqslant i \leqslant q, 1 \leqslant k<\infty\right\}, \quad a_{i k} \in N, a_{i 1}<a_{i 2}<\ldots$

$$
\left.A_{i}=\left\{a_{i 1}, a_{i 2}, \ldots\right\}, \quad A_{i j}=\left\{a_{i j}, a_{i, j+1}, \ldots\right\}, \quad \bar{A}_{i j}=A_{i}\right\urcorner A_{i j}
$$

According to (12), for $\varepsilon>0$ the number $j$ can be chosen so large that (13)

$$
\left.\delta\left(N A_{i j}\right\urcorner N \bar{A}_{i j}\right)<\varepsilon / q \text { for each } i=1, \ldots, q .
$$

- Denote by $\sigma_{j}$ the homogeneous system generated by

$$
\left\{S: S=a_{i k} S_{i}, 1 \leqslant i \leqslant q, 1 \leqslant k<j\right\} \text {. }
$$

By Theorem $3, \delta\left(\sigma_{j}\right)$ and $\lambda_{\sigma_{j}}$ exist and $\delta\left(\sigma_{j}\right)=\lambda_{\sigma_{j}}$. Hence there is a $\sigma_{j}$-free set $H_{j}$ with $\underline{\delta}\left(H_{j}\right)>\lambda_{a_{j}}-\varepsilon$. If $t_{i} \in S_{i}$ the set

is $\sigma$-free and, by (13),

$$
\left.\left.H_{j}^{\prime}=H_{j}\right\urcorner \bigcup_{i=1}^{q} t_{i}\left(N A_{i j}\right\urcorner N \bar{A}_{i j}\right)
$$

$$
\underline{\delta}(\sigma) \geqslant \underline{\delta}\left(H_{j}^{\prime}\right)>\dot{\lambda}_{\sigma_{j}}-2 \varepsilon
$$

Since $\sigma_{1} \subset \sigma_{2} \subset \ldots \subset \sigma$ the limit $\lim _{j \rightarrow \infty} \lambda_{\sigma_{j}}=\lambda$ exists and $\vec{\lambda}_{\sigma} \leqslant \lambda$. Now, on letting $j \rightarrow \infty$ and $\varepsilon \rightarrow 0$ in (14), we see that $\delta(\sigma)$ and $\lambda_{\sigma}$ exist and $\delta(\sigma)=\lambda_{\sigma}=\lambda$.

Finally, we are going to extend Lemma 6 by Lemma 5. Let $G=\left\{g_{1}, g_{2}, \ldots\right\}$ and $G_{j}=\left\{g_{j}, g_{j+1}, \ldots\right\}$. We shall say that $G$ has property $P$ if $\lim _{j \rightarrow \infty} \delta\left(N G_{j}\right)=0$.

THEORTM The homogeneous system $\sigma^{\prime}$ is generated by $\sigma_{0}^{\prime}=\left\{S_{1}, S_{2}, \ldots\right\}$. Let $G$ be a sequence with property $P$ and $M_{j}=$ $\bigcup\left\{S: S \in \sigma_{0}^{\prime}, S \cap N G_{j}=\emptyset\right\}$. If each set $M_{j}$ has property $P$, then for any homogeneous subsystem $\sigma \subset \sigma^{\prime}$ the densities $\delta(\sigma)$ and $\lambda_{\sigma}$ exist and coincide. by

Proof. If we denote by $\sigma_{j}$ and $\sigma_{j}^{\prime}$ the homogeneous systems generated. then $\sigma_{j} \subset \sigma_{j}^{\prime}$. Suppose (a) $\delta\left(\sigma_{j}\right)$ and $\lambda_{\sigma_{j}}$ exist and $\delta\left(\sigma_{j}\right)=\lambda_{\sigma_{j}}$ for each $j \in N$, then the existence of $\delta(\sigma)$ and $\lambda_{\sigma}$, and $\delta(\sigma)=\lambda_{\sigma}$ follow from Lemma 5 . If each set $M_{j}$ is finite, then (a) is true by Lemma 6 .

(b) Theorem 4 is true, if each set $M_{j}$ is finite. In the general casse (a) follows from (b) applied to $\sigma_{j}^{\prime}$.

\section{Examples}

EXAMPLE 1. Let $\sigma$ consist of the solutions in positive integers of the equation

$$
\text { (15) } \quad x_{1}+x_{2}+\ldots+x_{2 k}=2\left(y_{1}+y_{2}+\ldots+y_{2 k}\right) \text {. }
$$

Clearly, the interval $(n / 2, n]$ is $\sigma$-free. Therefore, $\underline{\tau}_{\sigma} \geqslant \frac{1}{2}$. We prove

$$
d(\sigma)=1 / r, \text { where } \quad r=\min \{z: z \in N, z \nmid 2 k\} \text {. }
$$

Obviously, the congruence class 1 modulo $r$ is $\sigma$-free. Hence $\underline{a}(\sigma) \geqslant 1 / r$. Let $A \subset N$ be $\sigma$-free. By equating some of the variables in (15) it follows that the equation

$$
x_{1}+x_{2}+\ldots+x_{j}=2\left(y_{1}+y_{2}+\ldots+y_{j}\right)
$$

has no solution in $A$, if $j$ divides $2 k$, thus especially for $j=1, \ldots, r-1$. For $x_{2}=y_{1}, x_{3}=y_{2}, \ldots, x_{j}=y_{j-1}$ the last equation becomes

$$
x_{1}=y_{1}+\ldots+y_{j-1}+2 y_{j} \quad(j=2,3, \ldots, r-1) .
$$

By (16), $x_{1}+x_{2}=2\left(y_{1}+y_{2}\right)$ has no solution in $A$. For $x_{1}=x_{2}$ this means that

$$
x_{1}=y_{1}+y_{2}
$$

is also unsolvable in $A$. Let $a \in A$. Substituting $y_{2}=y_{3}=\ldots=y_{j}=a$ in (17) and $y_{2}=a$ in (18) we see that none of the equations

$$
x_{1}=y_{1}+j a \quad(j=1,2, \ldots, r-1)
$$

has a solution in $A$. Hence $\bar{d}(A) \leqslant 1 / r$.

It would be interesting to know whether the logarithmic density $\delta(\sigma)$ exists for every homogeneous system defined by a linear equation.

EXAMPLI 2. We construct a finitely generated homogeneous system $\sigma$ with $\underline{d}(\sigma)<\bar{d}(\sigma)$. Suppose that $a$ is a positive integer not equal to 1 . Let $\sigma$ consist of all 3 -term geometric progressions of ratio $a, a^{2}, a^{3}$ or $a^{4}$. This system is generated by

$$
\left\{1, a, a^{2}\right\}, \quad\left\{1, a^{2}, a^{4}\right\}, \quad\left\{1, a^{3}, a^{6}\right\}, \quad\left\{1, a^{4}, a^{8}\right\} .
$$

We determine $\bar{d}(\sigma)=\tau_{\sigma}$ and $d(\sigma)=\lambda_{\sigma}$ according to the considerations to Theorem 1 and Theorem 3. By (4) and (11), we have

$$
\bar{d}(\sigma)=d(V) \sum_{r \in R} 1 / r, \quad \underline{d}(\sigma)=\alpha d(V),
$$


where $R$ is the set satisfying $R(n)=\tau_{\sigma}^{U}(n)$ and $\alpha=\lim \lambda_{\sigma}^{U}(n)$. We determine $\lambda_{\sigma}^{U}(n)=S^{*}(n)$ as indicated in the final part of the proof of Theorem 3 . Thus we obtain, by (19),

$$
\begin{gathered}
\bar{d}(\sigma)=\left(1-\frac{1}{a}\right)\left(1+\frac{1}{a}+\frac{1}{a^{3}}+\frac{1}{a^{4}}+\frac{1}{a^{8}}+\ldots\right), \\
\underline{d}(\sigma)=\left(1-\frac{1}{a}\right)\left(1+\frac{1}{a}+\frac{1}{a^{3}}+\frac{1}{a^{4}}\right) \sum_{j=0}^{\infty} \frac{1}{a^{9 j}}<\bar{d}(\sigma) .
\end{gathered}
$$

EXAMPLe 3. Denote by $C=\left\{c_{1}, c_{2}, \ldots\right\}$ the sequence of integers greater than 1 , which are a product of at most $k$ primes (multiple factors counted multiply). Define $S_{j}=\left\{1, c_{j}\right\}$, and let $\sigma$ be the homogeneous system generated by $\sigma_{0}=\left\{S_{1}, S_{2}, \ldots\right\}$.

By Lemma 1 and Lemma $2, \tau_{\sigma}$ exists, and there is a $\sigma$-free set $A$ with $\bar{d}(A)=\tau_{\sigma}$. Since $c_{j} \geqslant 2$ for each $j \in N$, we have $\tau_{\sigma} \geqslant \frac{1}{2}$. Let us prove

$$
\delta(\sigma)=\lambda_{\sigma}=\frac{1}{k+1} .
$$

Suppose that $A$ is a $\sigma$-free set in $[1, n]$ satisfying $A^{*}(n)=\lambda_{\sigma}(n)$. We sketchily follow the words of Halberstam and Roth ([3], pp. 24.6-249) for a proof of Behrend's theorem on primitive sequences.

$$
A^{*}(n)=\lambda_{\sigma}(n)=\frac{1}{n} \sum_{u \leqslant n} r(u)+O(1),
$$

where $r(u)$ is the number of divisors of $u$ belonging to $A$. Let $u$ be a product of $s(u)$ primes. According to de Bruijn, Tengbergen, and Kruyswijk [1], the set of divisors of $u$ can be completely divided into $\left(\begin{array}{c}s(u) \\ {[s(u) / 2]}\end{array}\right)$ disjoint symmetrical chains. A symmetrical chain of $m$ divisors cannot contain more than $\frac{m}{k+1}+1$ numbers of $A$. Therefore, if $d(u)$ is the number of divisors of $u$,

and, by (21)

$$
r(u) \leqslant \frac{d(u)}{l+1}+\left(\begin{array}{c}
s(u) \\
{[s(u) / 2]}
\end{array}\right)
$$

$$
\begin{gathered}
\lambda_{\sigma}(n) \leqslant \frac{1}{n(k+1)} \sum_{u \leqslant n} d(u)+O\left(\frac{1}{n} \sum_{u \leqslant n} \frac{d(u)}{(s(u))^{1 / 2}}\right) \\
\lambda_{\sigma}(n) \leqslant \frac{\log n}{n+1}+O\left(\frac{\log n}{(\log \log n)^{1 / 2}}\right) .
\end{gathered}
$$

On the other hand, if $A=\{a: a>1, s(a)=1 \bmod (k+1)\}$ then $A$ is $\sigma$-free, and it follows as before that

$$
A^{*}(n) \geqslant \frac{\log n}{k+1}+O\left(\frac{\log n}{(\log \log n)^{1 / 2}}\right) .
$$

By (22) and (23), we obtain (20). Note that the constants involved in the $O$-estimates of $(22)$ and $(23)$ can be chosen independent of $k$.

EXAMPLE 4. Let $\sigma$ consist of all $n$-term geometric progressions $(n \geqslant 3$, rational ratio). Systems of this kind have been investigated by Rankin [4] and by Riddell [5]. The system $\sigma$ is generated by

$$
\sigma_{0}=\left\{S: S=\left\{a^{n-1}, a^{n-2} b^{1}, a^{n-3} b^{2}, \ldots, b^{n-1}\right\}, a<b,(a, b)=1\right\} .
$$

Let $G=\left\{1^{n-1}, 2^{n-1}, 3^{n-1}, \ldots\right\}$. Since $\sum_{j} 1 / j^{n-1}$ converges, we have $\lim \bar{d}\left(N G_{j}\right)=0$. By Theorem 2, $\tau_{\sigma}$ exists, and from Lemma 1 follows

the existence of a $\dot{\sigma}$-free set $A$ with $\vec{d}(A)=\tau_{\sigma}$. Lemma 5 and Theorem 3 ensure the existence of $\delta(\sigma)$ and $\lambda_{\sigma}$. Moreover, $d(\sigma)=\delta(\sigma)=\lambda_{\sigma}$.

Suppose that $E \subset\{0\} \cup N$ is a set which does not contain an $n$-term arithmetic progression. Let $A$ consist of those positive integers which have in their unique prime factorization only exponents belonging to $E$. Then $A$ is $\sigma$-free, $d(A)$ exists, and

$$
\underline{d}(\sigma) \geqslant d(A)=\prod_{p}\left\{\left(1-\frac{1}{p}\right) \sum_{r \in Z^{\prime}} \frac{1}{p^{r}}\right\},
$$

where the product is taken over all primes. As in the proof of Theorem 3, it follows by induction that $\sum_{r=T} 1 / p^{r}$ is maximal if and only if $E$ is identical with the set $E_{n}$ defined by the following property:

$r \in E_{n}$ if and only if $\left(E_{n} \cap[0, r-1]\right) \cup\{r\}$ does not contain an $n$-term arithmetic progression $(r \in\{0\} \cup N)$.

The estimates of Rankin and Riddell obtained by (24) can be improved for $n \geqslant 4$, because they use a set $E \neq E_{n}$. If $n$ is a prime, then it follows from a paper of Scheid [6] that $E_{n}$ consists of the nonnegative integers, which have no digit $n-1$, when they are expressed in the scale of $n$. In this case we have

$$
\begin{aligned}
& \underline{a}(\sigma) \geqslant \prod_{p}\left\{\left(1-\frac{1}{p}\right) \prod_{k=0}^{\infty}\left(1+\frac{1}{p^{n^{k}}}+\frac{1}{p^{2 n^{k}}}+\ldots+\frac{1}{p^{(n-2) n^{k}}}\right)\right\}, \\
& \underline{d}(\sigma) \geqslant \prod_{p}\left\{\left(1-\frac{1}{p}\right) \prod_{l_{k=0}}^{\infty} \frac{1-\frac{1}{p^{(n-1) n^{k}}}}{1-\frac{1}{p^{n^{k}}}}\right\}=\frac{1}{\zeta(n-1)} \prod_{k=1}^{\infty} \frac{\zeta\left(n^{k}\right)}{\zeta\left((n-1) n^{k}\right)} .
\end{aligned}
$$


Now suppose that $\sigma^{\prime}$ consists of all 3 -term geometric progressions with integral ratio. We wish to show $d\left(\sigma^{\prime}\right)<\bar{d}\left(\sigma^{\prime}\right)$. Let $U$ be the sequence of positive integers which have no prime divisor different from 2 or 3 . Denote by $\sigma^{*}$ the system of those progressions in $\sigma^{\prime}$ which have ratio in $U$. It is not difficult to check that

$$
\max \left\{\sum_{u \in A} \frac{1}{u}: A \sigma^{*} \text {-free, } A \subset\left\{2^{r_{1}} 3^{r_{2}}: r_{1}, r_{2}=0,1,2\right\}\right\}=2 \text {. }
$$

Thus

$$
\alpha=\lim _{n \rightarrow \infty} \lambda_{\sigma^{*}}^{U}(n) \leqslant 2 \sum_{j=0}^{\infty} \frac{1}{2^{3 j}} \sum_{i=0}^{\infty} \frac{1}{3^{3 / c}}=\frac{8}{7} \frac{27}{13}
$$

and, by (11),

$$
\underline{d}\left(\sigma^{\prime}\right) \leqslant \underline{d}\left(\sigma^{*}\right) \leqslant\left(1-\frac{1}{2}\right)\left(1-\frac{1}{3}\right) \frac{8}{7} \frac{27}{13}=\frac{72}{91}=0.791 \ldots
$$

On the other hand the set

$$
\left(\frac{n}{32}, \frac{n}{27}\right) \cup\left(\frac{n}{24}, \frac{n}{12}\right) \cup\left(\frac{n}{9}, \frac{n}{8}\right) \cup\left(\frac{n}{4}, n\right)
$$

is $\sigma^{\prime}$-free in $[1, n]$. Hence

$$
\bar{d}\left(\sigma^{\prime}\right)=\tau_{\sigma^{\prime}} \geqslant \frac{5}{864}+\frac{1}{24}+\frac{1}{72}+\frac{3}{4}=\frac{701}{864}=0.811 \ldots
$$

\section{References}

[1] N. G. de Bruijn, C. E. Tengbergen and D. Kruyswijk, On the set of divisors of a number, Nieuw Arch. Wisk. II, 23 (1949-51), pp. 191-193.

[2] P. Erdös, Some extremal problems in combinatorial number theory, Mathematical essays dedicated to A. J. Macintyre, pp. 123-133, Ohio University Press, Athens 1970 .

[3] H. Halberstam and K. F. Roth, Sequences, Oxford University Press, 1966

4] R. A. Rankin, Sets of integers containing not more than a given number of terms in arithmetical progression, Proc. Royal Soc. Edinburgh Sect. A 65 (1962), pp. $332-344$.

[5] J. Rid dell, Sets of integers containing no n terms in geometrio progression, Glasgow Math. J. 10 (2) (1969), pp. 137-146.

[6] H. Scheid, Über arithmetische Progressionen der Länge p, J. Roino Angow. Math. 232 (1968), pp. 118-121.

\section{INSTITUT FUR MATHEMATIK} TECHNISCHE UNIVHRSTTAT

Clausthal, G. F. R.

XXXII (1977)

\section{Some remarks on Goldbach's problem}

by

AкIo FUJII (Tokyo)

In this paper we shall prove by a modification of Chen's work ([3]) that every sufficiently large even integer $x$ is written as a sum of a prime and a natural number which has at most one prime factor less than $x^{1089 / 2089}$.

1. Let $x$ be a large even integer. Let $G_{2}(x)$ be the number of primes $p \leqslant x$ such that $x-p$ has at most two prime factors. Chen ([3]) has proved. that

(1) $\quad G_{2}(x) \geqslant \frac{0.67 x O_{x}}{(\log x)^{2}}, \quad$ where $\quad O_{x}=\prod_{\substack{p \mid x \\ p>2}} \frac{p-1}{p-2} \prod_{p>2}\left(1-\frac{1}{(p-1)^{2}}\right)$.

In fact, if we put $G_{2}(x, I)$ - the number of primes $p \leqslant x$ such that $x-p$ is a prime or $x-p=p_{1} p_{2}$ with primes $p_{1}$ and $p_{2}$ satisfying $p_{1} \Phi$ and $p_{1} \leqslant p_{2}$, for a subset $I$ of $\left(1, x^{1 / 2}\right]$, he has proved that $G_{2}\left(x,\left(1, x^{1 / 10}\right]\right)$ $\geqslant 0.67 x C_{x} /(\log x)^{2}$. (Further Halberstam [6] or [7] has shown that 0.67 can be replaced by 0.689 .) Now we wish to maximize $I \subset\left(1, x^{1 / 2}\right]$ such that $G_{2}(x, I) \geqslant A x C_{x} /(\log x)^{2}$, where $A$ is some positive absolute constant. To study this we use the following mean value theorem which is similar to Bombieri's one.

LFMma 0. Assume that $M+N \ll x^{1 / 2}$. For an arbitrarity large constant $A$, there exist positive constants $B=B(A)$ and $E=E(A)$ such that if $M \gg(\log x)^{M}$, and $b(m) \ll(\log x)^{O}$. with some positive constant $C$ for any $m$ in $M<m \leqslant M+N$, then

$$
\begin{aligned}
\sum_{a \leqslant x^{1 / 2} /(\log x)^{B^{3}}} \max _{(a, d)=1} \max _{(I M+N)^{1+0}<y \leqslant x} & \left|\sum_{\substack{m=\pi(M+1 \\
(m, d)=1}}^{M+N} b(m)\left(\sum_{\substack{n \leqslant y / m \\
n=a m^{*}(d)}} A(n)-\frac{1}{\varphi(d)} \cdot \frac{y}{m}\right)\right| \\
& \leqslant x /(\log x)^{A}
\end{aligned}
$$

where 0 is an arbitrarity given positive number, $n \equiv a m^{*} \cdot(d)$ means $n \equiv a m^{*}(\bmod d)$, and $m^{*}$ satisfies $m m^{*} \equiv 1(d)$. 